\title{
Práticas Integrativas e Vivências em Arteterapia no Atendimento a Pacientes Oncológicos em Hospital Terciário
}

Integrative Practices and Experiences in Art Therapy in the Care of Oncology Patients in Tertiary Hospital

Prácticas Integrativas y Vivencias en Arteterapia en el Servicio a Pacientes Oncológicos en el Hospital Terciario

Maria Edna Bezerra da Silva ${ }^{1}$

Quitéria Silva do Nascimento Torres ${ }^{2}$

Thaline Barbosa e Silva ${ }^{3}$

Cleide de Sousa Araújo ${ }^{4}$

Tayse Lopes Alves ${ }^{5}$

\section{Resumo}

Objetivo: Relatar a experiência de vivencias de Arteterapia com grupo de pacientes com câncer, durante sessões de quimioterapia em um hospital universitário visando promover bemestar, elevar a autoestima, diminuir o estresse e a ansiedade, tornando o processo terapêutico mais humanizado.

Método: As sessões de Arteterapia foram realizadas entre os meses de abril a julho de 2017, contando com a participação de 30 (trinta) pessoas (pacientes e acompanhantes) cada sessão, com as seguintes etapas: apresentação da proposta de atividade, leitura de texto, conto ou história para sensibilização, seguida de algumas perguntas que indicassem $\mathrm{o}$ entendimento; orientação para a realização da atividade; fechamento com a partilha da experiência. Resultados: Foi possível perceber

${ }^{1}$ Professora Assistente da Faculdade de Medicina da Universidade Federal de Alagoas. Autora correspondente: Campus A. C. Simões. Av. Lourival Melo Mota, s/n. Tabuleiro dos Martins. 57072-900. Maceió, Al, Brasil.. E-mail: medna.pc@gmail.com

${ }^{2}$ Servidora do Núcleo de Saúde Pública da Universidade Federal de Alagoas. Especialista em Gestão do Trabalho em Saúde pela UFAL

${ }^{3}$ Arteterapeuta. Colaboradora da Sala de Cuidados da Universidade Federal de Alagoas

${ }^{4}$, Graduanda do Curso de Medicina da Universidade Federal de Alagoas

${ }^{5}$ Graduanda do Curso de Enfermagem da Universidade Federal de Alagoas

Recebido: Dez/2017 - Aceito: Abr2018.

Revist. Port.: Saúde e Sociedade. 2018;3(1):721-731. 
através das falas dos participantes, o bem-estar promovido e a melhora na ansiedade. A utilização da Arteterapia durante as sessões trouxe benefícios aos pacientes e seus acompanhantes, segundo os relatos dos mesmos, incentivando a continuidade da utilização desse recurso como forma de contribuir com a humanização da assistência prestada pelo serviço. Conclusão: São necessários outros estudos que avaliem os resultados e impactos para os usuários e seus acompanhantes, legitimando a introdução das práticas integrativas, no caso a Arteterapia, nos serviços de saúde.

\section{Descritores: Humanização da}

Assistência; Oncologia; Terapias Complementares.

\section{Abstract}

Objective: To report the experience of Art Therapy experiences with a group of cancer patients during chemotherapy sessions in a university hospital aimed at promoting wellbeing, elevating self-esteem, reducing stress and anxiety, making the therapeutic process more humanized. Method: Art therapy sessions were held between April and July 2017, with 30 participants (patients and companions) each session, with the following steps: presentation of the activity proposal, text reading, story or history for sensitization, followed by some questions that indicate the understanding; orientation for carrying out the activity; closure with the sharing of experience. Results: It was possible to perceive through the speeches of the participants, the promoted well-being and the improvement in the anxiety. The use of Art therapy during the sessions brought benefits to the patients and their companions, according to the reports of the same, encouraging the continuity of the use of this resource as a way to contribute to the humanization of the assistance provided by the service. Conclusions: Further studies are needed to evaluate the results and impacts for users and their companions, legitimizing the introduction of integrative practices, in the case of Art therapy, in health services.

Descriptors: Humanization of Assistance; Medical Oncology; Complementary Therapies.

\section{Resumen}


Objetivo: Relatar la experiencia de vivencias de Arteterapia con grupo de pacientes con cáncer, durante sesiones de quimioterapia en un hospital universitario para promover el bienestar, elevar la autoestima, disminuir el estrés y la ansiedad, haciendo el proceso terapéutico más humanizado. Método: Las sesiones de Arteterapia se realizaron entre los meses de abril a julio de 2017, contando con la participación de 30 (treinta) personas (pacientes y acompañantes) cada sesión, con las siguientes etapas: presentación de la propuesta de actividad, lectura de texto, cuento o historia para sensibilización, seguida de algunas preguntas que indicaran el entendimiento; orientación para la realización de la actividad; cierre con el compartir la experiencia. Resultados: Fue posible percibir a través de las palabras de los participantes, el bienestar promovido y la mejora en la ansiedad. La utilización de la Arteterapia durante las sesiones trajo beneficios a los pacientes y sus acompañantes, según los relatos de los mismos, incentivando la continuidad de la utilización de ese recurso como forma de contribuir con la humanización de la asistencia prestada por el servicio. Conclusiones: Se necesitan otros estudios que evalúen los resultados e impactos para los usuarios y sus acompañantes, legitimando la introducción de las prácticas integrativas, en el caso la Arteterapia, en los servicios de salud.

\section{Descriptores: Humanización de la Atención; Oncología Médica; Terapias}

\section{Complementarias.}

\section{Introdução}

O processo de implantação do Sistema Único de Saúde (SUS) tem contemplado diferentes propostas de mudanças no olhar sobre a saúde, com vista à integralidade da assistência e produção do cuidado, incluindo para isso, práticas integrativas em saúde como a Acupuntura, Homeopatia, Fitoterapia e Arteterapia.

O cuidar em saúde conquista uma dimensão maior e mais abrangente na atualidade, enfatizando não só as necessidades biológicas, mas também as necessidades emocionais, psicológicas, sociais e espirituais, fatores que contribuem para o surgimento de patologias no corpo físico ${ }^{(1)}$. Esse paradigma emergente é também chamado de holismo.

Esse campo de saberes holísticos e cuidados formam um quadro crescente de métodos diagnóstico-terapêuticos, 
com tecnologias leves, filosofias orientais e estratégias sensíveis de vivência corporal e autoconhecimento que contribuem para bem-estar físico e mental $^{(2)}$.

Instituídas pela Portaria $n^{\circ} 971$ do Ministério da Saúde em maio de 2006, as Práticas Integrativas e Complementares (PNPIC) foram constituídas em resposta a um movimento que se identifica com novos modos de aprender e praticar a saúde, já que essas práticas caracterizam-se pela interdisciplinaridade e linguagens singulares, que em geral se contrapõem à visão altamente tecnológica e biomédica de saúde que impera na sociedade de mercado.

De acordo com a Portaria, as Práticas Integrativas e Complementares (PICs) compreendem estimular os mecanismos naturais de prevenção de agravos e recuperação da saúde com ênfase na escuta acolhedora, no desenvolvimento do vínculo terapêutico e na integração do ser humano com o meio ambiente e a sociedade, além da visão ampliada do processo saúdedoença e a promoção global do cuidado humano, especialmente do autocuidado $^{(3)}$.

As PICs vêm apoiar, incorporar e implementar experiências que já são desenvolvidas por muitos municípios e estados entre as quais se destacam aquelas no âmbito da Medicina Tradicional Chinesa, como Massoterapia, Homeopatia Acupuntura, Reik, Reflexologia e Auriculoterapia ${ }^{(3)}$.

Os métodos utilizados preconizam elementos de origem natural e energia vibracional na prevenção de agravos, promoção, manutenção e recuperação da saúde ${ }^{(4)}$.

Visando atender às diretrizes da Organização Mundial de Saúde (OMS) e avançar na institucionalização das PICs no âmbito do SUS, em março de 2017, foi publicada a Portaria $N^{\circ} 849$, a qual ampliou o leque de terapias contempladas na Portaria 971/2006, incluindo a Arteterapia, Ayurveda, Biodança, Dança Circular, Meditação, Musicoterapia, Naturopatia, Osteopatia, Quiropraxia, Reflexoterapia, Reiki, Shantala e Terapia Comunitária Integrativa $^{(5)}$.

Este trabalho se propõe a relatar experiência realizada com a utilização da Arteterapia, uma das terapias reconhecidas na Portaria 849/2017, com acompanhantes e pacientes com câncer durante sessões de quimioterapia. A atividade foi realizada em um Centro de Oncologia de um Hospital Universitário, em parceria com o Núcleo de Saúde Pública (NUSP) da Faculdade de Medicina (FAMED) da 
Universidade Federal de Alagoas (UFAL).

As vivencias da Arteterapia objetivaram proporcionar bem-estar, diminuir o estresse e a ansiedade, tornando o processo mais leve, mais humanizado, bem como, buscando elevar a autoestima e promover autoconhecimento.

Ainda segundo a Portaria 849/2017, a Arterapia pode: estimular a expressão criativa, auxiliar no desenvolvimento motor, no raciocínio e no relacionamento afetivo. Através da arte é promovida a ressignificação dos conflitos, gerando a reorganização das próprias percepções, ampliando a percepção do individuo sobre si e o mundo. A arte é utilizada no cuidado à saúde com pessoas de todas as idades e por meio desta, a reflexão é estimulada sobre possibilidades de lidar de forma mais harmônica com o estresse $\mathrm{e}$ experiências traumáticas ${ }^{(5)}$.

A Arteterapia possibilita a expressão de conteúdos subjetivos, muitas vezes difíceis de serem verbalizados, sendo um instrumento valioso para a promoção da saúde e a qualidade de vida. A importância do processo expressivo se sobrepõe à preocupação com a estética do trabalho. Pode ser utilizada com diversos públicos e em contextos variados, podendo ser desenvolvida em trabalhos individuais ou em grupo. Esse meio de expressão utiliza uma variedade de modalidades expressivas e materiais tais como, pintura, modelagem, musica, poesia, dramatização e dança, devendo o profissional escolher a que melhor se adequa às características e necessidades do público com quem vai trabalhar ${ }^{(6)}$.

Vivencias de Arterapia tem sido cada vez mais utilizadas como prática do cuidado em saúde. $\mathrm{Na}$ área da saúde mental, o uso das expressões artísticas como forma terapêutica já é reconhecido pelos profissionais. Os benefícios percebidos têm chamado à atenção de cuidadores de outras áreas, estimulando a utilização da arte no processo psicoterapêutico. Além dos hospitais e clínicas psiquiátricas, estão sendo beneficiados hospitais gerais e clínicas de outras especialidades que atendem pessoas com doenças crônicodegenerativas $^{(7)}$.

Acredita-se que mediante a interpretação e a reflexão das vivências na relação terapêutica, a pessoa vai se apropriando dos seus próprios conteúdos, conhecendo a si mesma e se tornando assim, sujeito ativo do processo terapêutico ${ }^{(8)}$.

Várias experiências da utilização da Arteterapia com pacientes oncológicos gerando benefícios 
emocionais, físicos e psicológicos tem sido relatadas e apontam para a consolidação do seu uso. A partir de estudos baseados na prática clínica, evidenciou-se que oportunizar aos pacientes com câncer a vivência de processo artístico pode favorecer a ressignificação da própria vida, uma vez que facilita encontrar formas de lidar com a realidade vivenciada no processo de doença e tratamento ${ }^{(7)}$.

Em experiência para administração do ócio com pacientes oncológicos hospitalizados, foi utilizada a técnica de colagem, a qual permitiu deduzir comportamentos e sentimentos dos pacientes através da interpretação artística pelo teste de cores de Lüscher ${ }^{(9)}$. Possibilitou-se que os estudantes envolvidos na experiência pudessem observar os pacientes por um novo ângulo, vendo uma riqueza de expressões, sentimentos e emoções, no que se refere à situação que vivenciavam, bem como, em relação a sua vida e lembranças, apontando a Arteterapia como estratégia para atender às necessidades emocionais e afetivas dos pacientes ${ }^{(9)}$.

Um outro trabalho relata o uso da Arteterapia com um grupo de pacientes e seus acompanhantes em um hospital universitário, onde foram realizadas atividades tais como: pintura, recorte, desenho, colagem, visualização criativa e cromoterapia ${ }^{(10)}$. Considerou-se que o trabalho realizado foi importante, pois as técnicas de Arteterapia proporcionaram autoconhecimento, resgate da autoestima e sensação de bem-estar por meio do relaxamento, além de promover felicidade e reduzir o estresse ${ }^{(9)}$. Outros benefícios também foram relatados, como: promover a alegria, visão de futuro e desejo de mudanças; ocupação das horas ociosas; facilitar o diálogo entre paciente e acompanhante; e melhoria na sua qualidade de vida ${ }^{(10)}$.

Aliado ao desenvolvimento crescente da Medicina no tratamento de diversas doenças crônicas se faz necessário aliar aos cuidados com a dimensão física dos pacientes, a oferta de práticas terapêuticas que os contemplem em sua totalidade psíquica e que se adaptem à rotina das instituições hospitalares com vistas à humanização da assistência à saúde ${ }^{(7)}$.

Outra publicação relata uma experiência inspiradora utilizando a Terapia Expressiva através de várias ações dentro de um programa de extensão que integra ensino, pesquisa e assistência e visa contribuir para humanização e integralidade do cuidado, desenvolver o cuidado de si entre profissionais de saúde, aprimorar a 
qualidade do cuidado aos usuários, contribuir para a formação em saúde e cultura e produzir evidências sobre a eficácia da Terapia Expressiva ${ }^{(11)}$.

O desenvolvimento do trabalho, objeto deste relato iniciou-se a partir da parceria entre o Núcleo de Saúde Pública (NUSP), da Faculdade de Medicina da Universidade Federal de Alagoas e o Centro de Oncologia CACON do Hospital Universitário Professor Alberto Antunes. A proposta inicial foi trazer as práticas integrativas e complementares já desenvolvidas pelo Projeto de Extensão Sala de Cuidados Professor Antônio Piranema, na UFAL, como parte das atividades do projeto "Práticas Integrativas: fazem mais pela vida", desenvolvido por alunas do estágio de Serviço Social no serviço.

Durante os meses de abril a julho de 2017, semanalmente, foram realizadas diversas práticas, tendo como público os pacientes e acompanhantes da sala de espera e da sala de quimioterapia, tais como: Dança Circular, Reiki, Reflexologia, Auriculoterapia, Arteterapia, Calatonia, Cura Reconectiva e Massagem.

Após o encerramento do estágio, em virtude dos resultados positivos das experiências, as práticas foram reiniciadas no mês de julho, com periodicidade quinzenal, mas com esforço conjunto dos profissionais e instituições envolvidas para a elaboração/estruturação de um projeto que possibilite não somente a continuidade dos cuidados, como a sua ampliação e institucionalização.

\section{Método}

A experiência deste relato referese às sessões de Arteterapia realizadas com pacientes e acompanhantes durante o tratamento com a quimioterapia nos meses de abril, maio e julho de 2017, contando com a participação de uma média de 30 (trinta) pessoas (pacientes e acompanhantes) em cada sessão. Foram realizadas três sessões com duração de duas horas cada, com as seguintes etapas: apresentação da proposta de atividade; leitura de texto, conto ou história para sensibilização, seguida de algumas perguntas que evidenciassem o entendimento; orientação para a realização da atividade; e fechamento com a partilha da experiência.

Durante as sessões, utilizou-se um fundo musical para auxiliar na concentração e relaxamento. As modalidades utilizadas foram técnica utilizando costura e técnica de colagem com revistas.

Utilizando fios podemos simbolicamente tecer a trama da nossa 
vida, organizando nosso mundo interior e exterior, esse processo quando trazido à consciência possibilita construir ou desconstruir a nossa própria história ${ }^{(12)}$. Entre outras indicações, a técnica de bordado é recomendada para desenvolvimento da autoestima, perseverança, paciência e capacidade de lidar com erros e acertos.

Ao se referir à técnica de colagem, especificamente utilizando revistas, a mesma é indicada para mobilizar conteúdos internos, recordar sentimentos e despertar emoções, uma vez que as imagens são repletas de simbolismos, permitindo que a pessoa manifeste seu inconsciente sem se expressar diretamente ${ }^{(12)}$. Seu uso é recomendado para trabalhar com pessoas que necessitem unir partes, colar pedaços internamente, para início de atividades terapêuticas, no trabalho com pessoas inibidas ou que alegam não ter habilidades artísticas ${ }^{(12)}$.

No primeiro momento, foi feita a proposta da confecção de um coração em tecido, costurado à mão, com enchimento. A partir do texto "Coração Remendado", estimulou-se a reflexão sobre os sentimentos e emoções presentes (De que o meu coração está cheio?). E, ao confeccionar o coração, preenchê-lo com os sentimentos e emoções positivos desejados $(\mathrm{O}$ que eu quero que preencha meu coração?).

Em outra sessão, foi proposta a confecção de uma flor em tecido. Utilizou-se o texto "Se as coisas fossem mães" de Sylvia Orthof, onde os participantes puderam externar sentimentos relacionados às mães ou às pessoas que fizeram esse papel em sua vida. Para a atividade de colagem, foi usado o texto "Vende-se" de Olavo Bilac. A proposta foi refletir sobre si mesmo, as próprias qualidades e características e expressá-las na forma de um anúncio pessoal, através de colagem e utilizando revistas.

\section{Resultados e Discussão}

Os resultados obtidos referem-se aos registros de relatos dos pacientes e acompanhantes durante a etapa da partilha, bem como, observação e registro do comportamento dos envolvidos durante 0 processo. Participaram das ações, um total aproximado de 90 pessoas.

No que se refere ao comportamento, tanto os profissionais do setor de quimioterapia quanto os pacientes e acompanhantes foram receptivos ao desenvolvimento das atividades propostas. Poucas pessoas deixaram de participar do trabalho. Em 
uma situação, observou-se que, mesmo sem participar diretamente, a paciente interagiu com sua acompanhante tornando o produto uma expressão da dupla. Em outra, o paciente não pode participar de todas as etapas da atividade, em virtude de ter começado a sessão de quimioterapia mais cedo. Um paciente recebeu o material enquanto aguardava a sessão e expôs que não sabia fazer o trabalho proposto, e assim que iniciou a quimioterapia colocou o material de lado e não participou.

Essas situações foram compreendidas como reação da situação física ou emocional dos pacientes, sendo suas vontades respeitadas pelas facilitadoras. Em situações onde a pessoa não demonstra interesse em participar de atividades expressivas, mesmo sendo estimuladas, orienta-se respeitar a vontade do usuário $\mathrm{e}$ aguardar para fazer uma nova abordagem em outro momento ${ }^{(8)}$.

$\mathrm{Na}$ fase da partilha, os participantes verbalizaram como foi a atividade para eles:

Um negócio desse bota a gente pra frente, ajuda a gente a superar (Participante 1)

Boa dinâmica para quem está acompanhando e quem tá com problema (Participante 2)
A tristeza vai embora, (Participante3)

Foi bom para expressar o que passa, (Participante 4)

Foi produtivo (Participante 5)

Ajuda a mascarar a situação, participante 6 .

Foi muito lindo! Amei.

(Participante 7)

Palavras bonitas, música suave... Não percebi o tempo passar, (Participante 8)

Pudemos perceber nessas falas que as atividades colaboraram para tornar o tempo menos ocioso, diminuir a ansiedade, promover bem-estar e melhorar o estado de ânimo. Uma paciente comparou os nove meses de tratamento com a espera para (re) nascer, trazendo esperança de vitória e vida nova para si e sua família. Um acompanhante lembrou da experiência de um membro de sua família que utilizou a expressão através da colagem como ajuda para vencer a depressão.

Em relação à expressão artística, os participantes refletiram sobre $\mathrm{o}$ trabalho realizado e através de suas interpretações, foi possível perceber que o processo possibilitou: promoção da autoestima e autoconhecimento; resgate de sentimentos e valores positivos; relaxamento e sensação de bem-estar; manifestação de sentimentos de esperança, amor, paz, felicidade, superação, alegria; um (re) encontro 
com qualidades pessoais esquecidas; reflexão sobre a importância da família e amigos; resgate do desejo de fazer atividades de que gostam, como estar com a família, fazer tricô, cozinhar, fotografar, gostar de flores, natureza, assistir filme, ler bons livros, ouvir boa música, ir a shows e exposições, praticar esportes, o cuidado pessoal (vestimenta, alimentação saudável, maquiagem, exercício físico, arrumarse, cuidado com saúde bucal).

O cuidado humanizado potencializa as relações humanas para a promoção de sentimentos de prazer, confiança e respeito mútuo, restabelecendo assim, o desejo de viver e cuidar de si mesmo ${ }^{(10)}$.

Faz-se necessário a realização de mais estudos que avaliem os efeitos da Arteterapia para os pacientes e acompanhantes como forma de incentivar a inserção dessa terapia nos serviços de saúde. A realização de ações de Terapia Expressiva em instituições hospitalares públicas é um desafio. A produção de evidências sobre sua eficácia demanda a formação de uma equipe interdisciplinar, recursos, abertura de espaços institucionais e o desenvolvimento de pesquisas $^{(11)}$.

\section{Conclusão}

O trabalho desenvolvido indica que a utilização da Arteterapia durante as sessões de quimioterapia pode trazer benefícios aos pacientes e seus acompanhantes, promovendo bem-estar e relaxamento, tornando o tempo de espera mais produtivo, colaborando para a melhora do estado de ânimo, estimulando o autoconhecimento e aumento da autoestima, entre outros. Acreditamos na importância da continuidade da utilização desse recurso como forma de colaborar com a humanização da assistência e na atenção integral aos pacientes.

A experiência com Arteterapia no Centro de Oncologia do hospital em questão ainda é incipiente. No entanto, os resultados positivos observados, aliados à dedicação e interesse de profissionais das instituições envolvidas e voluntários que acreditam na importância dessa prática, servem de motivação para continuar empreendendo esforços para estruturação de um projeto que possibilite sua institucionalização, possibilitando não somente a continuidade dos cuidados, como sua ampliação. 


\section{Referências}

1. Galli BSK, Scararti M, Diehl AA, Lunkes TJ, Rojahn D, Schoeninger D. Saúde e equilíbrio através das terapias integrativas: Relato de experiência. Enfermagem (Rio Grande do Sul) [periódico na Internet]. 2012 [citado 2017 ago. 25]; 8(8): 245-55. Disponível em: http://revistas.fw.uri.br/index.php/r evistadeenfermagem/article/view/491/8 97.

2. Aroucha EBL. Práticas Integrativas e Complementares: o interesse em formação dos profissionais da estratégia de Saúde da Família - Recife-PE. Monografia (Residência Multiprofissional em Saúde Coletiva) Departamento de Saúde Coletiva, Centro de Pesquisas Aggeu Magalhães, Fundação Oswaldo Cruz; 2010. Disponível em: http://www.cpqam.fiocr uz.br/bibpdf/2012aroucha-ebl.pdf.

3. Ministério da Saúde (BR). Secretaria-Executiva. Portaria $n^{\circ} 971$ de 3 maio de 2006: Política Nacional de Práticas Integrativas e Complementares (PNPIC) no Sistema Único de Saúde. Brasília: Ministério da Saúde; 2006.

4. Alvim TAN, Pereira VML, Martins FAP, Rohr VR, Pereira MDR. Práticas integrativas e complementares no cuidado: Aplicabilidade e implicações para enfermagem. $17^{\circ}$ Seminário Nacional de Pesquisa em Enfermagem; 2013. Disponível em: www.abenevent os.com.br-anais_senpe/17senpe/pdf/007 Opr.pdf.

5. Ministério da Saúde (BR). Secretaria-Executiva. Portaria $n^{\circ} 849$, de 27 de março de 2017: Inclui Novas Práticas Integrativas na Política Nacional de Práticas Integrativas e Complementares (PNPIC) no Sistema Único de Saúde. Brasília: Ministério da Saúde; 2017.
6. Reis AC. Arteterapia: a arte como instrumento no trabalho do Psicólogo. Arteterapia (Brasília) [periódico na Internet]. 2014 [citado 2017 ago. 22]; 34(1): 142-57. Disponível em: http://www.scielo.br/scielo.php?script=s ci_arttext\&pid=S1414-9893201400010 0011 .

7. Vasconcellos EA, Giglio JS. Introdução da arte na psicoterapia: enfoque clínico e hospitalar. Estud. Psicol. (Campinas) [periódico na Internet]. 2007 [citado 2017 ago. 25]; 24(3): 375-83. Disponível em:http://www.scielo.br/scielo.php?scri $\mathrm{pt}=$ sci_arttext\&pid=S0103-166X20070 $00300009 \& \operatorname{lng}=$ en $\&$ nrm $=$ iso\&tlng $=$ pt.

8. Coqueiro NF, Vieira FRR, Freitas MMC. Art therapy as a therapeutic tool in mental health. Enfermagem (Fortaleza) [periódico na Internet]. 2010 [citado 2017 ago. 29]; 23(6): 859-62. Disponível em: http://www.scielo.br/p df/ape/v23n6/en_22.pdf.

9. Barbosa ICFJ, Santos MCL, Leitão GCM. Arteterapia na assistência de enfermagem em oncologia: produções, expressões e sentidos entre pacientes e estudantes de graduação. Enfermagem (Rio de Janeiro) [periódico na Internet]. 2007 [citado 2017 set. 11]; 11(2): 22733. Disponível em: http://www .scielo.br/scielo.php?pid=S1414-81452 007000200007\&script=sci_abstract\&tln $\mathrm{g}=\mathrm{pt}$.

10. D'Alencar ÉR, Souza ÂMA, Araújo TS, Beserra FM, Lima MMR, Gomes AF. Arteterapia no enfrentamento do câncer. Enfermagem (Fortaleza) [periódico na Internet]. 2013 [citado 2017 set. 16]; 14(6):1241-8. Disponível em: http://www.periodico s.ufc.br/rene/article/view/3752. 
11. Vianna D, Mendes AA, Claro LL, Silva AN, Bucci DA, Rocha VS, et al. Terapia Expressiva: veículo de cuidado integral num hospital universitário. Naturologia e Terapias Complementares (Santa Catarina) [periódico na Internet]. 2012 [citado 2017 set. 24]; 1(1):101-6. Disponível em: http://portaldeperiodicos .unisul.br/index.php/CNTC/article/view $/ 1016$.

12. Araújo MM, Santos RB.

Arteterapia Transpessoal. Recife:

Comunigraf; 2010. 